Nuclear Physics A386 (1982) 79-100

(C) North-Holland Publishing Company

\title{
TOWARDS A BETTER PARAMETRISATION OF SKYRME-LIKE EFFECTIVE FORCES: A CRITICAL STUDY OF THE SkM FORCE $\dagger$
}

\author{
J. BARTEL and P. QUENTIN* \\ Institut Laue-Langevin, BP 156X, 38042 Grenoble Cedex, France \\ and
}

M. BRACK, C. GUET ${ }^{* *}$ and H.-B. HÅKANSSON

Inst. für Theor. Physik, Universität Regensburg, Regensburg, Fed. Rep. Germany

Received 15 February 1982

\begin{abstract}
Static nuclear properties associated with a Skyrme-like force in the SkM parametrisation have been extensively studied for both spherical and deformed nuclei through Hartree-Fock plus BCS calculations. These calculations include in particular the determination of the ${ }^{240} \mathrm{Pu}$ fission barrier up to the second saddle point. The validity of some currently used approximations to the Hartree-Fock approach (self-consistent Strutinsky approach, expectation value method and twostep iterative method) has also been assessed. The results of the microscopic calculations have been systematically compared to the corresponding self-consistent results obtained within the extended Thomas-Fermi framework. Such semiclassical calculations also allow a proper characterisation of the SkM force surface properties (in the liquid drop or droplet model sense). Whereas ground-state radii and multipole moments are found in excellent agreement with experimental data, binding energies are systematically too high and fission barriers are significantly too low. These two defects are shown to be correlated through the too low surface tension of the force. A modified parametrisation is discussed which heals both these defects while keeping intact the good reproduction of other properties. This constitutes a first step in the direction of current efforts to determine a better parametrisation of Skyrme-like forces.
\end{abstract}

\section{Introduction}

Over the last ten years, Hartree-Fock (HF)+BCS calculations using phenomenological density-dependent effective forces like Skyrme or gaussian forces have been found very satisfactory in reproducing static and low energy dynamical nuclear properties [see e.g. ref. ${ }^{1}$ )]. One of the most simple, yet very successful parametrisations is the one referred to as the Skyrme SIII effective force ${ }^{2}$ ). It has appeared increasingly clear, however, that the adjustment of this type of force still needs to

+ Work partially supported by Deutsche Forschunsgemeinschaft (DFG).

* Present address: Laboratoire de Physique Théorique, Université de Bordeaux-I, Domaine du Haut-Vigneau, 33170 Gradignan, France.

** Present address: Centre d'Etudes Nucléaires de Grenoble, DRF/CPN, BP 85X, 38041 Grenoble Cedex, France. 
be considerably improved in order to guarantee a better description of: (i) the isovector electric dipole (E1) giant resonance, (ii) the isoscalar electric monopole (E0) giant resonance, and (iii) actinide fission barrier heights. Incidentally, one would also like to control singlet-even properties of the force in order to secure correct $(T=1)$ pairing matrix elements. A Skyrme force called SkM fulfilling the requirements (i) and (ii) has been recently proposed ${ }^{3}$ ). As a part of the general project of finding a better parametrisation of Skyrme forces, it is one aim of this paper to study the actinide fission barrier heights [requirement (iii)] as obtained with the SkM force. In what follows we will not discuss the pairing properties of the SkM force which would probably be inadequate since they have not entered the fitting procedure in any direct way.

Before studying the static fission barriers obtained with the SkM force, one should first investigate in some detail its static ground-state properties. Since the authors of ref. ${ }^{3}$ ) have mainly based their fit on nuclear matter saturation properties and on giant E0 and E1 modes, it remains necessary therefore to calculate both spherical and deformed finite nuclei within the $\mathrm{HF}$ framework [including an approximate treatment of pairing correlations as described in ref. $\left.{ }^{4}\right)$ ].

The self-consistent calculations for deformed nuclei are lengthy and costly. How time consuming they are, depends on the imposed symmetries. The calculation of spherically symmetric solutions is very fast. Axially symmetric solutions, usually obtained through a truncated basis expansion (which appears to be the only reasonable procedure for heavy nuclei so far), require a considerable amount of computing time essentially because of the necessity of correctly minimising the energy with respect to the basis parameters. Releasing the left-right symmetry would increase slightly the numerical effort, whereas it is almost impossible in the present state of the art to think of systematic, fully self-consistent calculations for heavy nuclei releasing the axial symmetry. It is therefore necessary, particularly in view of making a fit of effective forces, to develop handy yet secure approximation schemes. This aspect of the problem will be thoroughly studied in the present work.

Three approximations will be considered in this paper: (i) the standard shell correction method ${ }^{5-10}$ ) in its self-consistent version ${ }^{11,12}$ ), (ii) the expectation value method ${ }^{13,14}$ ), and (iii) the two step iterative method ${ }^{15-17}$ ). These approximations have already been tested for the SIII Skyrme force ${ }^{12,14,16}$ ). We will extend here these studies to the SkM force. The use of self-consistent semiclassical densities (either in the framework of the partial $\hbar$ resummation method ${ }^{18-20}$ ) or in the extended Thomas-Fermi (ETF) model ${ }^{21-24}$ ) would improve considerably the accuracy of the above-listed approximations. This is why we will also present some results of semiclassical calculations with the SkM force in the ETF approach.

The paper will be organised as follows: In sect. 2 we will present saturation, surface and symmetry properties of the SkM force for infinite nuclear matter and using spherically symmetric HF results for magic nuclei. Sect. 3 will be devoted to the study of deformation properties of this force (including the ${ }^{240} \mathrm{Pu}$ fission barrier). 
Finally we will conclude in sect. 4 , putting the present study in the more general perspective of finding a satisfactory Skyrme force parametrisation by indicating a possible way of improving the surface properties of the SkM force.

\section{Saturation, surface and symmetry properties of spherical nuclei}

The SkM force parameters have been adjusted in such a way as to yield correct saturation properties in infinite isoscalar nuclear matter. The incompressibility $\boldsymbol{K}_{\infty}$ as well as the symmetry and surface properties have been taken care of by requiring a fair reproduction of isoscalar E0, isovector E0 and isovector E1 giant resonance properties in a fluid dynamical lagrangian approach. In this section we will recall briefly the infinite nuclear matter properties of the SkM force, discuss its symmetry and surface properties and present some HF results for spherically symmetric finite nuclei calculated with this force.

For the most general central Skyrme force [with the notation of e.g. ref. ${ }^{25}$ )]

$$
\begin{aligned}
v_{\text {central }}\left(r_{1}, r_{2}\right)= & t_{0}\left(1+x_{0} P_{\sigma}\right) \delta(r)+t_{1}\left(1+x_{1} P_{\sigma}\right)\left[\delta(r) k^{2}+k^{\prime 2} \delta(r)\right] \\
& +t_{2}\left(1+x_{2} P_{\sigma}\right) k^{\prime} \delta(r) k+t_{3}\left(1+x_{3} P_{\sigma}\right) \rho^{\alpha}(\boldsymbol{R}) \delta(\boldsymbol{r})
\end{aligned}
$$

the binding energy per particle $(E / A)$, the incompressibility $\left(K_{\infty}\right)$ and the volume symmetry energy $(J)$ are expressed as (with $\left.\beta=\frac{3}{5}\left(\frac{3}{2} \pi^{2}\right)^{2 / 3}\right)$ :

$$
\begin{gathered}
\frac{E}{A}=\frac{\hbar^{2}}{2 m} \beta \rho^{2 / 3}+\frac{3}{8} t_{0} \rho+\frac{1}{4}\left[\frac{3}{4} t_{1}+t_{2}\left(x_{2}+\frac{5}{4}\right)\right] \beta \rho^{5 / 3}+\frac{1}{16} t_{3} \rho^{\alpha+1}, \\
K_{\infty}=2 \frac{\hbar^{2}}{2 m} \beta \rho^{2 / 3}+\frac{9}{4} t_{0} \rho+5\left[\frac{3}{4} t_{1}+t_{2}\left(x_{2}+\frac{5}{4}\right)\right] \beta \rho^{5 / 3}+\frac{9}{16} t_{3}(\alpha+1)\left(\alpha+\frac{2}{3}\right) \rho^{\alpha+1}, \\
J=\frac{5}{9} \frac{\hbar^{2}}{2 m} \beta \rho^{2 / 3}-\frac{t_{0}}{4}\left(x_{0}+\frac{1}{2}\right) \rho-\frac{5}{24}\left[t_{1} x_{1}-\frac{1}{3} t_{2}\left(4+5 x_{2}\right)\right] \beta \rho^{5 / 3}-\frac{t_{3}}{48}\left(2 x_{3}+1\right) \rho^{\alpha+1}
\end{gathered}
$$

in terms of the isoscalar density $\rho=\rho_{\mathrm{n}}+\rho_{\mathrm{p}}$ determined by the saturation condition $\partial / \partial \rho(E / A)=0$. The effective mass $m^{*}$ in isoscalar nuclear matter is given by

$$
m^{*}=m\left\{1+\frac{1}{4} \frac{2 m}{\hbar^{2}} \rho\left[\frac{3}{4} t_{1}+t_{2}\left(x_{2}+\frac{5}{4}\right)\right]\right\}^{-1} .
$$

The SkM values of $E / A, K_{\infty}, J, \rho$ and $m^{*} / m$ are listed in table 1 where they are compared with those obtained with the SIII force ${ }^{2}$ ). Whereas the two forces lead to almost identical saturation energies, the SkM force yields significantly more compact nuclear matter (i.e., a larger equilibrium density) than the SIII force, which is favourable since SIII systematically overestimates charge radii in both spherical and deformed nuclei [see table 5 of ref. ${ }^{1}$ )]. The incompressibility which was bound to be at least of the order of $300 \mathrm{MeV}$ with linearly density-dependent effective 
forces ${ }^{2}$ ) is fixed for the SkM force to a value consistent with experimental isoscalar E0 giant resonance data ${ }^{26}$ ), as discussed in ref. ${ }^{27}$ ). The symmetry volume coefficients $J$ are roughly comparable for the two forces. They are smaller by $\sim 30 \%$ than the values obtained within Brückner and local density approximation calculations ${ }^{28}$ ) and those deduced from the more recent droplet model fits to binding energies ${ }^{29}$ ).

The surface properties of a force may be characterised by the parameters $a_{\mathrm{S}}, \kappa_{\mathrm{S}}$ in an extended liquid drop model fit of average binding energies $\bar{E}$ of given nuclei $(N, Z)$ to the expression:

$$
\bar{E}=a_{\mathrm{v}}\left[1-\kappa \mathrm{v}\left(\frac{N-Z}{A}\right)^{2}\right] A+a_{\mathrm{s}}\left[1-\kappa \mathrm{s}\left(\frac{N-Z}{A}\right)^{2}\right] A^{2 / 3}+\cdots+E_{\text {Coul }},
$$

where $a_{\mathrm{V}}=E / A$ and $a_{\mathrm{V}} \kappa_{\mathrm{V}}=J$, the dots representing possible lower-order terms (e.g. a curvature term proportional to $A^{1 / 3}$ ). Of course, it would make no sense to fit eq. (6) to the exact HF binding energies due to the presence of shell effects. However, in a semiclassical model [like the ETF energy density method which was recently developed ${ }^{23,24}$ ) using Skyrme forces] the average part of the binding energy is obtained and can be fitted to eq. (6). In this respect let us note that in order to get unique values for $a_{\mathrm{s}}$ and $\kappa_{\mathrm{s}}$, curvature and constant terms must be included in the least-square fit procedure and values of $A$ up to $\geqslant 5000$ must be taken into account ${ }^{32}$ ). The resulting values of $a_{\mathrm{s}}, \kappa_{\mathrm{s}}$ are given in table 1 . When comparing the results obtained with the SIII and SkM forces, it appears that for the SkM force, both isoscalar and isovector contributions lead to a decrease of the surface tension compared with the SIII force. For ${ }^{240} \mathrm{Pu}$, for instance, the effective surface tension $a_{\mathrm{s}}\left[1-\kappa_{\mathrm{S}}(\{N-Z\} / A)^{2}\right]$ is decreased by $\sim 16 \%$. Since one expects from the values of $\rho$ in table 1 an increase in radii of $\sim 3 \%$, one deduces that the fissility parameter for ${ }^{240} \mathrm{Pu}$ will be increased by $13 \%$ thus leading to a significant reduction of the fission barrier. This is indeed the case, as will be shown in sect. 3 .

TABLE 1

Infinite nuclear matter and surface properties of the SIII, SkM and modified SkM forces; see text for the notation

\begin{tabular}{lccc}
\hline & SIII & SkM & SkM (modified) \\
\hline$E / A(\mathrm{MeV})$ & -15.86 & -15.78 & -15.78 \\
$K_{\infty}(\mathrm{MeV})$ & 355.4 & 216.7 & 216.7 \\
$m^{*} / m$ & 0.76 & 0.79 & 0.79 \\
$J(\mathrm{MeV})$ & 28.16 & 30.75 & 30.03 \\
$\rho\left(\mathrm{fm}^{-3}\right)$ & 0.1453 & 0.1603 & 0.1603 \\
$a_{\mathrm{S}}(\mathrm{MeV})$ & 18.13 & 16.85 & 17.51 \\
$\kappa_{\mathrm{S}}$ & 1.84 & 3.60 & 3.74 \\
\hline
\end{tabular}

The surface parameters have been determined from the calculated semiclassical (ETF) binding energies (see also ref. ${ }^{32}$ ), especially for the parameter $\kappa_{\mathrm{s}}$ ]. The modified SkM force (last column) is discussed in sect. 4. 
A systematic way of determining the coefficients of eq. (6) other than by a least-square fit is to make use of the leptodermous expansion ${ }^{30}$ ). This approach has recently been revisited in particular for Skyrme forces $\left.{ }^{31,32}\right)^{\dagger}$. The surface coefficients $a_{\mathrm{S}}$ and $\kappa_{\mathrm{S}}$ can be exactly obtained from semi-infinite (asymmetric) nuclear matter calculations. The lower order coefficients, however, can be correctly obtained only from a leptodermous expansion for finite nuclei, due to the nontrivial $\mathrm{N}$ - and $\mathrm{Z}$-dependence of the nucleon densities inside the nucleus ${ }^{32}$ ). Care has to be taken also of the self-consistency effect of the Coulomb interaction on the nuclear energy in eq. (6). It can be accounted for by an explicit "Coulomb redistribution energy" term $C_{2} Z^{2} A^{1 / 3}$ (+ lower-order terms) as in the droplet model ${ }^{30}$ ), if the coefficient $C_{2}$ is suitably adjusted. If no such term is added to eq. (6), the coefficients $a_{\mathrm{S}}, \kappa_{\mathrm{s}}, \ldots$ have to be appreciably changed. (The surface energy, $a_{\mathrm{S}}$, for instance would have to be increased by approximately $1 \mathrm{MeV}$.) For a more explicit discussion of the leptodermous expansion obtained within the ETF energy density method and, in particular, a detailed comparison with the droplet model, we refer to ref. ${ }^{32}$ ).

Let us now turn to finite nuclei. For nine shell or sub-shell closed nuclei $\left({ }^{16} \mathrm{O}\right.$, ${ }^{40} \mathrm{Ca},{ }^{48} \mathrm{Ca},{ }^{56} \mathrm{Ni},{ }^{90} \mathrm{Zr},{ }^{114} \mathrm{Sn},{ }^{132} \mathrm{Sn},{ }^{140} \mathrm{Ce},{ }^{208} \mathrm{~Pb}$ ) we have obtained spherically symmetric HF solutions, using the numerical procedure (and code) discussed in ref. $^{33}$ ). Pairing correlations (with constant matrix element $G$ ) were included in the standard approximate BCS way ${ }^{4}$ ). (Non zero gaps $\Delta_{n}$ or $\Delta_{\mathrm{p}}$ were obtained for the nuclei ${ }^{90} \mathrm{Zr},{ }^{114} \mathrm{Sn},{ }^{140} \mathrm{Ce}$.) A Coulomb exchange contribution in the Slater approximation has been included as well as the one-body part of the standard centre-of-mass correlation (i.e. $-T / A$, where $T$ is the kinetic energy). The resulting binding energies are displayed in table 2 . They are in reasonable agreement with experimental data ${ }^{34}$ ), however the fit is not as good as the one obtained with the SIII force. In fact, the SkM force leads to a systematic overbinding. One might suspect that this feature could be due to the inclusion of the Slater-approximated Coulomb exchange energy or of the one-body c.m. correcting term. However, the variation with $A$ of both corrections is inconsistent with the observed binding energy excess as shown in fig. 1. As a matter of fact, the deficiency can be traced back to a surface effect corresponding to an underestimation of the surface tension parameter $a_{\mathrm{S}}$ by roughly $\sim 0.6 \mathrm{MeV}$.

In table 2 we have also reported the ETF binding energies. As already found with other forces ${ }^{24}$ ), this approach leads for the SkM force to a slight overbinding ( $\sim 5-10 \mathrm{MeV}$ for heavy nuclei when a proper shell correction energy is added).

Charge radii obtained in both HF and ETF calculations are compared with experimental data in table 3 . The HF densities $\rho_{\mathrm{p}}(r)$ corresponding to point-like particles have been convoluted with a proton form factor to yield the charge

${ }^{+}$Note that ref. ${ }^{31}$ ) contains some numerical errors which are corrected in ref. ${ }^{32}$ ). 
TABLE 2

Experimental ( $\left.B^{\text {exp }}\right)$, Hartree-Fock $\left(B^{\mathrm{HF}}\right)$ and extended ThomasFermi $\left(B^{\mathrm{ETF}}\right)$ binding energies (in MeV)

\begin{tabular}{llrl}
\hline & $B^{\text {exp }}$ & $B^{\text {HF }}$ & $B^{\text {ETF }}$ \\
\hline${ }^{16} \mathrm{O}$ & 127.6 & 131.5 & 130.1 \\
${ }^{40} \mathrm{Ca}$ & 342.1 & 347.9 & 351.4 \\
${ }^{48} \mathrm{Ca}$ & 416.0 & 428.1 & 427.7 \\
${ }^{56} \mathrm{Ni}$ & 484.0 & 495.1 & 490.8 \\
${ }^{90} \mathrm{Zr}$ & 783.9 & 796.6 & 796.5 \\
${ }^{114} \mathrm{Sn}$ & 971.6 & 982.6 & 987.1 \\
${ }^{132} \mathrm{Sn}$ & 1102.7 & 1123.7 & 1111.1 \\
${ }^{140} \mathrm{Ce}$ & 1172.7 & 1188.0 & 1186.0 \\
${ }^{166} \mathrm{Er}^{(*)}$ & 1351.6 & 1362.9 & \\
${ }^{208} \mathrm{~Pb}^{240} \mathrm{Pu}^{(*)}$ & 1636.5 & 1652.7 & 1639.7 \\
\hline${ }^{2 *}$ & 1813.5 & 1822.5 & \\
\hline
\end{tabular}

The nuclei marked by a star are deformed in their ground states. For a discussion of the HF calculation for the latter nuclei, see below in the text and table 6. All other nuclei have been assumed to be spherically symmetric. Pairing correlations have been included in $B^{\mathrm{HF}}$ for protons in ${ }^{90} \mathrm{Zr}\left(\Delta_{\mathrm{p}}=1.7 \mathrm{MeV}\right)$ and ${ }^{140} \mathrm{Ce}\left(\Delta_{\mathrm{p}}=1.9 \mathrm{MeV}\right)$, for neutrons in ${ }^{114} \mathrm{Sn}\left(\Delta_{\mathrm{n}}=2.0 \mathrm{MeV}\right)$.

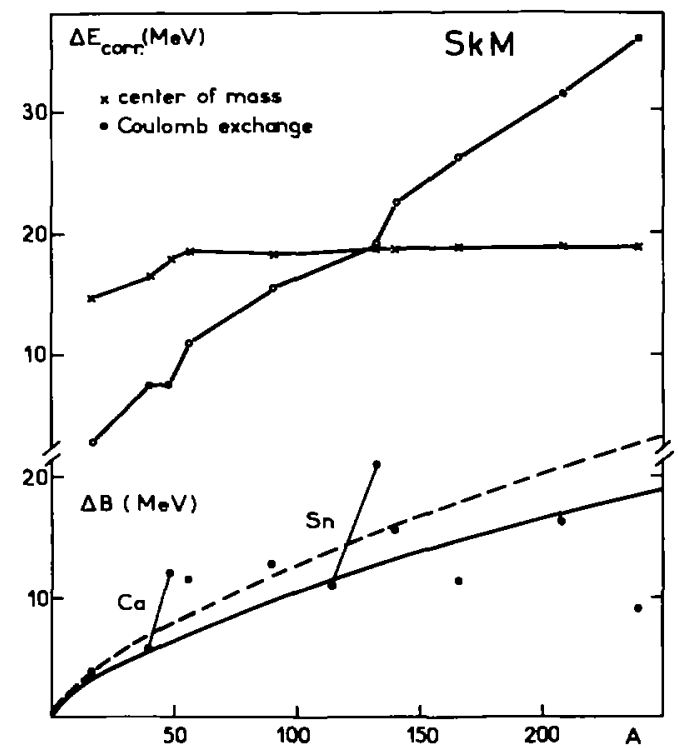

Fig. 1. Binding energy excess $\Delta B$, as obtained with the $S k M$ force for various spherical and deformed nuclei calculated within the Hartree-Fock plus BCS approach. For $\mathrm{Ca}$ and $\mathrm{Sn}$ nuclei, the straight lines suggest the isotopic structure of this excess. The results of a least square fit of $\Delta B$ to $\alpha A^{2 / 3}$ (namely with $\left.\alpha=\sum_{i}\left(A_{i}^{2 / 3} \Delta B_{l}\right) / \sum_{i} A_{i}^{4 / 3}\right)$ are also plotted as a solid (dashed) line when including deformed and spherical (only spherical) nuclei. For the sake of comparison, the variation with the nuclear number $A$ of the one-body c.m. correction and approximate Slater Coulomb exchange energies are also displayed at the top of the figure. 
TABLE 3

Comparison of experimental rms charge radii $r_{c}^{\exp }$ (in $\mathrm{fm}$ ) with various theoretical extimates

\begin{tabular}{lllllllllll}
\hline & $r_{\mathrm{c}}^{\text {exp }}$ & $r_{\mathrm{c}}^{\mathrm{HFC}}$ & $r_{\mathrm{c}}^{\mathrm{HF}}$ & $r_{\mathrm{c}}^{\mathrm{ETF}}$ & $\left(r_{n}-r_{p}\right)^{\text {HF }}$ & $\left(r_{n}-r_{p}\right)^{\mathrm{ETF}}$ & $\alpha_{n}$ & \multicolumn{1}{c}{$\alpha_{\mathrm{p}}$} & $\rho_{\mathrm{p}}^{0}$ & $\rho^{0}$ \\
\hline${ }^{16} \mathrm{O}$ & 2.73 & 2.71 & 2.79 & 2.75 & -0.03 & -0.02 & 0.440 & 0.444 & 0.0811 & 0.1641 \\
${ }^{40} \mathrm{Ca}$ & 3.49 & 3.46 & 3.50 & 3.45 & -0.04 & -0.04 & 0.450 & 0.458 & 0.0801 & 0.1629 \\
${ }^{48} \mathrm{Ca}$ & 3.48 & 3.46 & 3.52 & 3.52 & 0.16 & 0.17 & 0.490 & 0.433 & 0.0716 & 0.1627 \\
${ }^{56} \mathrm{Ni}$ & 3.75 & & 3.75 & 3.79 & -0.05 & -0.05 & 0.454 & 0.463 & 0.0790 & 0.1612 \\
${ }^{90} \mathrm{Zr}$ & 4.27 & 4.24 & 4.28 & 4.27 & 0.06 & 0.08 & 0.481 & 0.445 & 0.0717 & 0.1588 \\
${ }^{114} \mathrm{Sn}$ & & & 4.58 & 4.59 & 0.10 & 0.08 & 0.485 & 0.444 & 0.0699 & 0.1568 \\
${ }^{132} \mathrm{Sn}$ & & & 4.71 & 4.73 & 0.24 & 0.25 & 0.530 & 0.430 & 0.0627 & 0.1555 \\
${ }^{140} \mathrm{Ce}$ & $4.88^{*}$ & & 4.88 & 4.87 & 0.12 & 0.14 & 0.501 & 0.437 & 0.0662 & 0.1551 \\
${ }^{208} \mathrm{~Pb}$ & 5.50 & 5.47 & 5.49 & 5.52 & 0.18 & 0.17 & 0.518 & 0.432 & 0.0616 & 0.1510 \\
\hline
\end{tabular}

The HF values $r_{\mathrm{c}}^{\mathrm{HF}}$ include the effect of a proton charge form factor. For some nuclei these values have been further corrected for c.m., electromagnetic spin-orbit and neutron charge form factor effects, yielding the values $r_{\mathrm{c}}^{\mathrm{HFC}}$. The HF neutron-proton rms radius difference $\left(r_{\mathrm{n}}-r_{\mathrm{p}}\right)^{\mathrm{HF}}$ is listed. Both $r_{\mathrm{c}}$ and $\left(r_{n}-r_{p}\right)$ are also given in the ETF approximation. We give the diffuseness parameters $\alpha_{n}, \alpha_{p}$ and the central densities $\rho_{\mathrm{p}}^{0}$ and $\rho^{0}=\rho_{\mathrm{n}}^{0}+\rho_{\mathrm{p}}^{0}$ of the semiclassical ETF neutron (n) and proton $(\mathrm{p})$ densities (parametrised as Fermi functions). The starred experimental radius $\left({ }^{140} \mathrm{Ce}\right)$ corresponds to natural cerium containing $88.5 \%$ of ${ }^{140} \mathrm{Ce}$.

distribution. For some nuclei the HF values have been corrected for the spurious c.m. motion ${ }^{35}$ ) by:

$$
\Delta r=-\frac{1.1 \mathrm{fm}}{A}
$$

For the same nuclei we have also included the correction for the electromagnetic spin-orbit and neutron charge distribution effects ${ }^{36}$ ), evaluated ${ }^{17}$ ) for the same nuclei and with similar forces. As a general result one finds that the agreement between experimental ${ }^{37.38}$ ) and theoretical rms radii is very good and, in fact, far better than what was obtained with the SIII force ${ }^{2}$ ). It is, in particular, gratifying to see that the anomalous ${ }^{40} \mathrm{Ca}-{ }^{48} \mathrm{Ca}$ isotopic shift is qualitatively well reproduced, which was hardly the case for usual Skyrme forces, except for those (Ska, Skb) fitted by Köhler ${ }^{39}$ ). The charge radii obtained in the ETF calculations reproduce very well the (uncorrected) HF results except when well-marked shell effects are present, such as in the calcium isotopes. The differences between neutron and proton rms radii (for point-like nucleons) are also listed in table 3. The ETF results almost exactly reproduce the HF differences. From the ETF densities (parametrised as Fermi functions) one can study the variation of the central densities $\rho_{\mathrm{n}}^{0}, \rho_{\mathrm{p}}^{0}$ and diffuseness parameters $\alpha_{n}, \alpha_{p}$ as functions of $A$. Note that isotopic effects are rather important for the neutron diffuseness parameter $\alpha_{n}$, whereas the proton diffuseness $\alpha_{p}$ stays roughly constant, as already found in refs. ${ }^{24,40}$ ).

The proton and neutron single-particle energy spectra for the spherical nuclei ${ }^{90} \mathrm{Zr}$ and ${ }^{208} \mathrm{~Pb}$ are shown in figs. 2 and 3 . They agree qualitatively well with the 


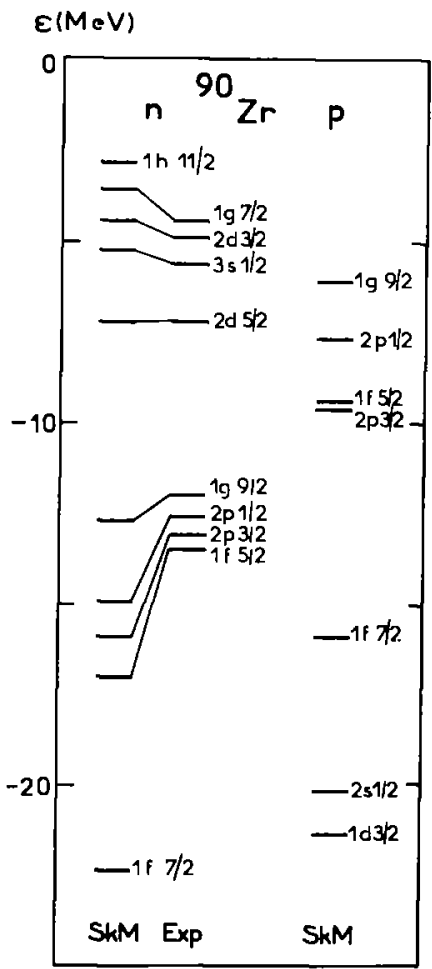

Fig. 2. Neutron and proton single-particle energies in the ${ }^{90} \mathrm{Zr}$ nucleus. Experimental energies have been taken from the compilation of ref. ${ }^{33}$ ). Pairing correlations have been included for protons (leading to $\Delta_{\mathrm{p}}=1.7 \mathrm{MeV}$ ).

displayed experimental data. One notes however a significant decompression of the HF spectra as compared with the experimental ones. Such an effect, which is only apparent for medium heavy and heavy nuclei, is well explained in terms of the interplay between single-particle motion and low-lying collective vibrations [see e.g. the discussion in ref. $\left.{ }^{1}\right)$ ]. It will be further discussed in subsect. 3.2 when presenting single-particle spectra of deformed nuclei.

\section{Deformation and fission properties}

\subsection{HARTREE-FOCK + BCS CALCULATIONS WITH AN EXTERNAL CONSTRAINT}

To calculate nuclear deformation energy surfaces, the constrained Hartree-Fock equations are solved by expanding the single-particle wave functions onto eigenstates of an axially symmetrical harmonic oscillator ${ }^{4,41}$ ). Special attention has to be paid to the convergence of the necessarily truncated expansion ${ }^{42}$ ) and to the best choice of the oscillator parameters at each deformation. The latter is imposed 


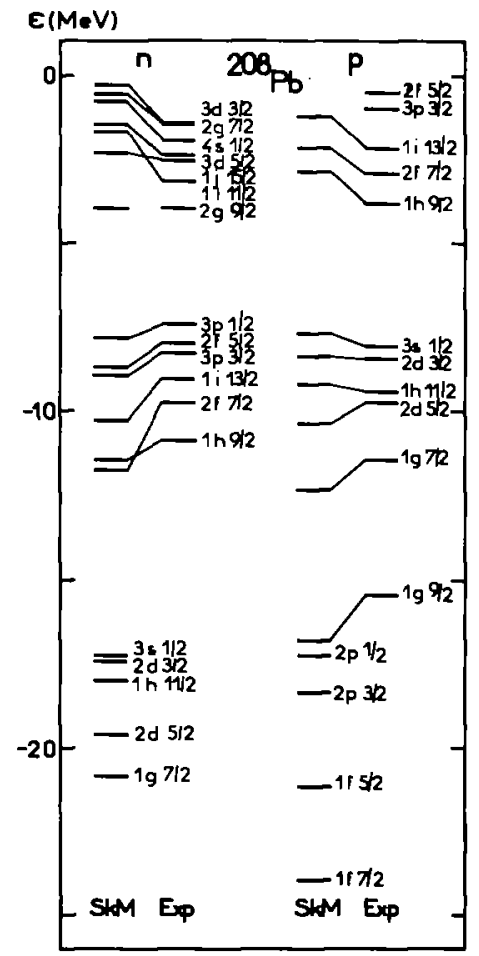

Fig. 3. Same as fig. 2 for the ${ }^{208} \mathrm{~Pb}$ nucleus.

by adding external constraints on one or several multipole moments to the HF hamiltonian [see ref. ${ }^{42}$ ) for an extensive study]. In the calculations reported here we will only consider a quadrupole constraint.

Pairing correlations should in principle be included through a Hartree-FockBogoliubov (HFB) approach. It has been shown ${ }^{43}$ ), however, that for static properties a self-consistent $\mathrm{HF}+\mathrm{BCS}$ method is a very accurate approximation to the full HFB treatment. In our calculations we have, for the sake of simplicity, included pairing correlations in an approximate $\mathrm{HF}+\mathrm{BCS}$ scheme [as formulated e.g. in ref. $\left.\left.{ }^{4}\right)\right]$ using a constant antisymmetrised pairing matrix element

$$
G_{i j}=\langle i \bar{i}|\nu| \widetilde{j}\rangle=-G .
$$

Since $G$ is not directly known empirically, rather than taking $G$ as a parameter we use the "uniform gap method" proposed in ref. ${ }^{6}$ ). At each deformation the value of $G$ is fixed by imposing the average pairing gap $\bar{\Delta}$ empirically known to vary as $\tilde{\Delta} \approx 12 \mathrm{MeV} / \sqrt{ } A$. Solving the BCS equations with the corresponding $G$, one obtains pairing gaps $\Delta_{\mathrm{n}}$ and $\Delta_{\mathrm{p}}$ in good agreement with the experimentally observed odd-even mass differences for all nuclei under consideration. 


\subsection{GROUND-STATE DEFORMATION PROPERTIES}

In this subsection we will briefly present some calculated ground-state properties of two well-deformed nuclei, namely ${ }^{166} \mathrm{Er}$ as a representative of the rare earth nuclei and ${ }^{240} \mathrm{Pu}$ as a typical actinide nucleus. Charge quadrupole, hexadecapole moments and rms radii of ${ }^{166} \mathrm{Er}$ calculated with the SkM force, are listed in table 4 together with available experimental data ${ }^{44,45}$ ). They are also compared with the results obtained using the Skyrme interaction SIII [ref. $\left.{ }^{46}\right)$ ] and Gogny's D1 force ${ }^{47}$ ), or those obtained ${ }^{48}$ ) within the density matrix expansion approach ${ }^{49.50}$ ).

\section{TABLE 4}

Comparison of charge rms radii, quadrupole and hexadecapole moments of ${ }^{166} \mathrm{Er}$, obtained in selfconsistent Hartree-Fock (present work) and shell-correction calculations ${ }^{54.56}$ ) with experimental data, $\left.{ }^{2}\right)$ : ref. ${ }^{44}$ ), ( ): ref. ${ }^{45}$ )

\begin{tabular}{|c|c|c|c|c|c|c|c|}
\hline & Exp & SkM & SIII & DME & D1 & Ref. ${ }^{54}$ ) & Ref. ${ }^{56}$ ) \\
\hline$r^{c}(\mathrm{fm})$ & $\left.5.238^{a}\right)$ & 5.246 & 5.331 & 5.262 & & & \\
\hline$Q_{2}^{\mathrm{c}}(\mathrm{b})$ & $\left.7.63 \pm 0.03^{b}\right)$ & 7.68 & 7.79 & 7.78 & 7.60 & 7.26 & 7.08 \\
\hline$Q_{4}^{c}\left(b^{2}\right)$ & $\left\{\begin{array}{l}\left.0.22_{-0.16}^{+0}\right) \\
\left.0.30^{*}\right)\end{array}\right.$ & 0.24 & 0.33 & 0.28 & & 0.27 & 0.31 \\
\hline
\end{tabular}

A similar comparison is made in table 5 for the ground and fission isomeric states of ${ }^{240} \mathrm{Pu}$ [see refs. ${ }^{51-53}$ ) for relevant experimental data]. For both nuclei we give also in tables 4,5 the results of Strutinsky shell-correction calculations using various phenomenological single-particle potentials ${ }^{8.54-57}$ ). It can be concluded that the SkM force yields moments which are in very good agreement with experimental data, this agreement being generally better than what was obtained with other forces (and comparable to the best phenomenological results).

TABLE 5

Same as table 4 for ${ }^{240} \mathrm{Pu}$ both in ground (g.s.) and fission isomeric state (f.i.s.)

\begin{tabular}{|c|c|c|c|c|c|c|}
\hline & $\operatorname{Exp}$ & Skm & SIII & Ref. ${ }^{55}$ ) & Ref. ${ }^{56}$ ) & Ref. ${ }^{57}$ ) \\
\hline$r^{c}(\mathbf{f m})$ & & 5.886 & 5.952 & & & \\
\hline$Q_{2}^{\text {g.s. }}(\mathrm{b})$ & $\left.11.58 \pm 0.08^{\mathrm{a}}\right)$ & 11.9 & 11.1 & 11.9 & 10.3 & 11.3 \\
\hline$Q_{4}^{\text {g.A. }}\left(\mathrm{b}^{2}\right)$ & $\left.1.15 \pm 0.28^{b}\right)$ & 1.2 & 1.1 & 1.1 & 1.0 & 1.4 \\
\hline$Q_{2}^{\text {f.t.s }}(\mathrm{b})$ & $\left.36 \pm 4^{\mathrm{c}}\right)$ & 34.0 & 32.7 & 38.2 & & \\
\hline
\end{tabular}

Experimental results are extracted from ${ }^{a}$ ): ref. $^{51}$ ), ${ }^{b}$ ): ref. ${ }^{52}$ ) and ${ }^{c}$ ): ref. ${ }^{53}$ ); results of shell-correction calculations are from refs. ${ }^{55-57}$ ).

In table 6 the binding energies of the ${ }^{166} \mathrm{Er}$ and ${ }^{240} \mathrm{Pu}$ nuclei are compared with experimental data ${ }^{34}$ ). They have been corrected as usual ${ }^{1}$ ) for truncation effects due to the expansion on a finite oscillator basis, for one-body c.m. motion effects and for spurious rotational energies. For the latter correction, we have taken the values obtained in ref. $\left.{ }^{58}\right)$ with the SIII force, i.e. with usual notation $\left\langle J^{2}\right\rangle / 2 \not{\Phi}=2.44$ 
TABLE 6

Ground state energies (in MeV) calculated with the SkM force

\begin{tabular}{llllll}
\hline & $E_{\mathrm{HF}+\mathrm{BCS}}$ & $\Delta E_{\text {trunc }}$ & $\left.\Delta E_{\mathrm{rot}}{ }^{2}\right)$ & $E_{\text {corrected }}$ & $E_{\text {exp }}{ }^{\text {b') }}$ \\
\hline${ }^{166} \mathrm{Er}$ & -1357.9 & -2.6 & -2.4 & -1362.9 & -1351.6 \\
${ }^{240} \mathrm{Pu}$ & -1817.4 & -3.3 & -1.9 & -1822.6 & -1813.5 \\
\hline
\end{tabular}

Before comparing the $\mathrm{HF}+\mathrm{BCS}$ energies $\left(E_{\mathrm{HF}+\mathrm{BCs}}\right)$ to experimental energies [ $\left.{ }^{\mathrm{b}}\right)$ : ref. $\left.{ }^{34}\right)$ ], we have subtracted truncation $\left(\Delta E_{\text {trunc }}\right)$ and spurious rotation $\left(\Delta E_{\text {rot }}\right)$ energies to yield corrected calculated values $\left(E_{\text {corrected }}\right)$. The truncation correction, assumed to be deformation independent and corresponding to 11 shells (13 shells) for ${ }^{166} \mathrm{Er}\left({ }^{240} \mathrm{Pu}\right.$ ), has been evaluated for spherically symmetric nuclei. The spurious rotation energy has been taken from the evaluation made in "): ref. ${ }^{58}$ ) for the SIII force. Note that the truncation error is significantly smaller with the SkM force than was the case for the SIII force which might be due to the different values of the incompressibility.

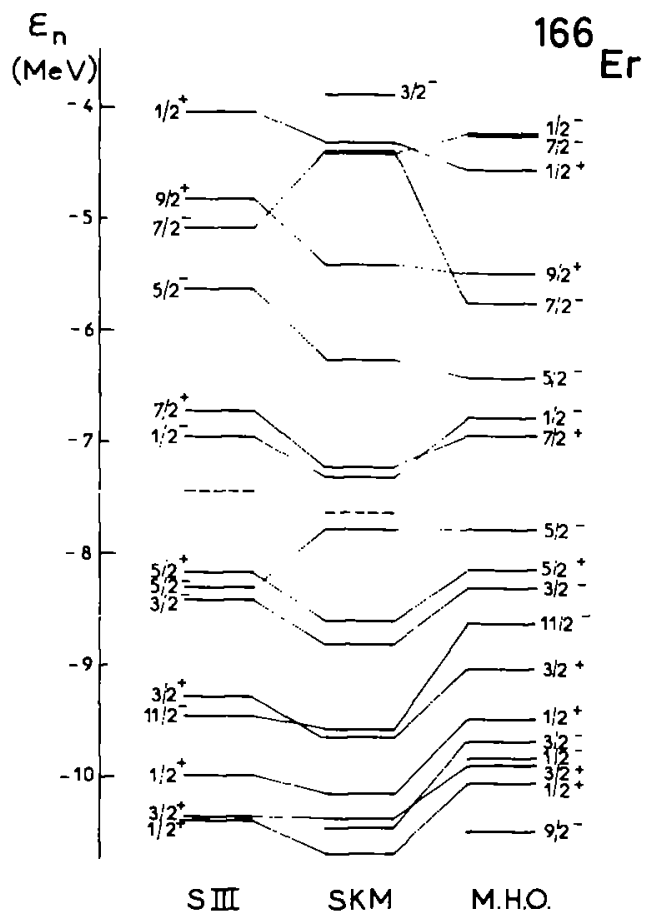

Fig. 4. Neutron single-particle energies corresponding to the equilibrium deformed mean field obtained for ${ }^{166} \mathrm{Er}$ with the SIII and SkM forces. Levels are labelled by their $\Omega^{*}$ values (with usual notation). Modified harmonic oscillator (MHO) estimates ${ }^{59}$ ) are also plotted, using the standard scaling $\hbar \dot{\omega}=$ $41 A^{-1 / 3} \mathrm{MeV}$ and choosing the MHO energy origin by matching some MHO and SkM levels located near the Fermi sea surface (here a $\frac{5}{2}-$ level). The Fermi energies obtained in self-consistent calculations are represented as dashed lines. 


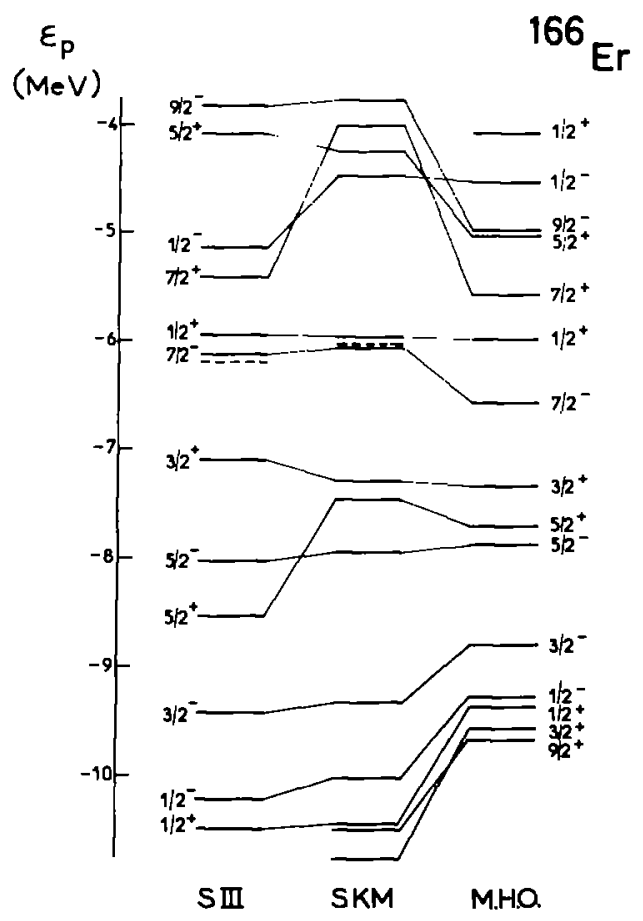

Fig. 5. Same as fig. 4 for proton levels.

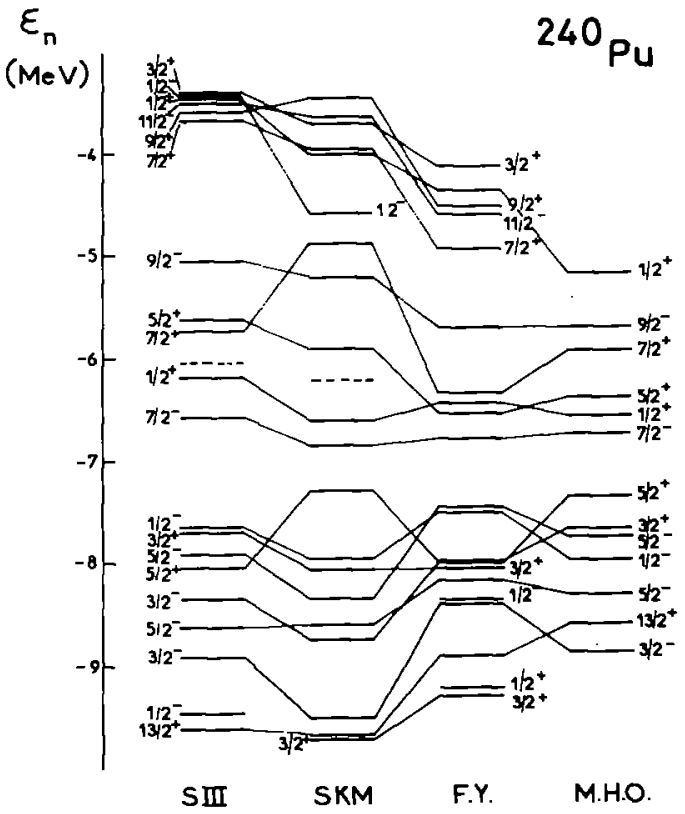

Fig. 6. Same as fig. 3 for the equilibrium deformed ${ }^{240} \mathrm{Pu}$ solution. Both folded Yukawa (FY) ${ }^{56}$ ) and modified harmonic oscillator (MHO) ${ }^{59}$ ) estimates are given. 
(1.89) $\mathrm{MeV}$ for ${ }^{166} \mathrm{Er}\left({ }^{240} \mathrm{Pu}\right)$. For these nuclei, as was the case for spherical nuclei (see sect. 2), the SkM force leads to a slight overbinding as shown in fig. 1.

The question arises whether the HF + BCS scheme is also capable of giving the right deformation behaviour of single-particle energies. We display in figs. 4-7 the self-consistent neutron and proton single-particle level spectra in the ground states of ${ }^{166} \mathrm{Er}$ and ${ }^{240} \mathrm{Pu}$ obtained with SkM and SIII forces. They are compared with the single-particle level schemes given by the Nilsson model ${ }^{59}$ ) and by the folded Yukawa potential approach of Möller, Nilsson and $\mathrm{Nix}^{56}$ ). The spectra obtained with the two different Skyrme forces in the HF + BCS approximation are rather similar. Moreover, they agree in all cases fairly well with the spectra of phenomenological shell-model potentials. For ${ }^{166} \mathrm{Er}$, and to a lesser extent for ${ }^{240} \mathrm{Pu}$, one notices an almost complete disappearance of the decompression in the HF spherical spectra (with respect to experimental data) noted in sect. 2 . This has been already discussed for the SIII force in ref. ${ }^{1}$ ). It is related to the coupling of individual degrees of freedom to low-lying collective vibrations which turns out to be more important in spherical than in deformed nuclei. The relevant parameter for the single-particle level density near the Fermi energy is the nuclear matter effective mass $m^{*}$ which is comparable for both SIII and SkM forces ( 0.76 and 0.79 , in nucleonic mass units, see table 1). It is therefore not surprising that the SkM force yields also a correct level density. The more detailed agreement of deformed single-particle energies with phenomenologically adjusted spectra is indeed more significant. It partly reflects the adequacy of the spin-orbit force strength. An even more stringent test of the deformation behaviour of single-particle energies is provided by spectroscopic

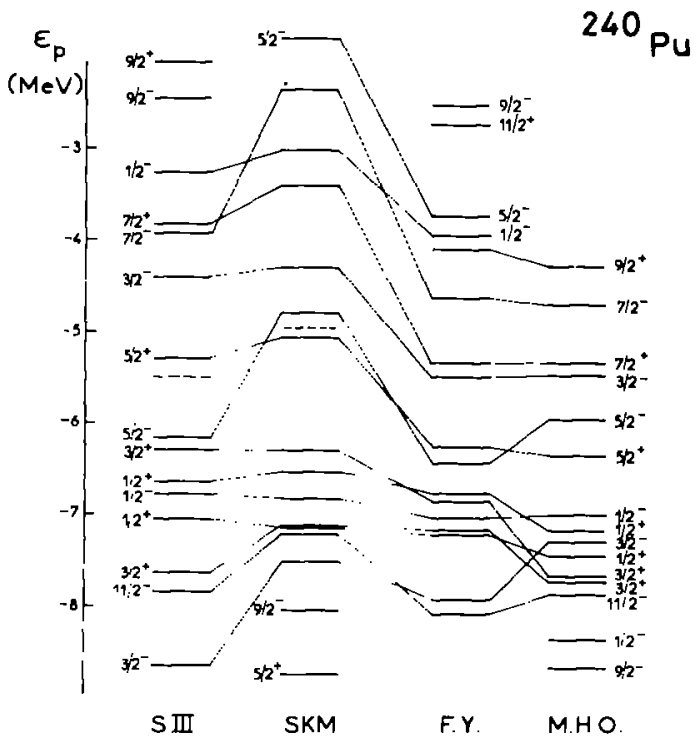

Fig. 7. Same as fig. 6 for proton levels. 
properties of odd nuclei fission isomeric states. For the ${ }^{239} \mathrm{Pu}$ nucleus the latter have been studied in ref. ${ }^{60}$ ) in the rotor-plus-one quasiparticle model. The correct location of the $\frac{9}{2}-$ and the neighbouring $\frac{5}{2}{ }^{+}$single-particle states obtained with the SIII force within this approach has been found essential for obtaining a very nice agreement with the observed states of the corresponding rotational bands. It has been found that the SkM neutron spectrum is indeed very close to what was obtained with SIII and we expect therefore the SkM force to yield similarly good spectroscopic properties for those very exotic nuclear states.

\subsection{THE ${ }^{240} \mathrm{Pu}$ FISSION BARRIER}

Self-consistent calculations of actinide fission barriers were performed in 1973 by the Orsay group ${ }^{61.62}$ ) using the SIII Skyrme force in the HF + BCS approach. The important qualitative features of experimentally observed fission barriers were reproduced by these calculations: existence of a double-humped fission barrier and correct deformation properties of both ground and fission isomeric states. However, even after applying various corrections due to truncation effects, to spurious translational and rotational energies and to the undue imposition of axial and left-right reflexion symmetries, the fission barrier heights were found significantly too high ${ }^{\dagger}$.

Shortly after these calculations, rather similar results have been obtained ${ }^{64}$ ) within the closely related phenomenological $K$-matrix approach. Full HartreeFock-Bogoliubov calculations ${ }^{63}$ ) have been achieved in 1979 making use of the gaussian effective interaction D1 due to Gogny ${ }^{43}$ ). Again these calculations yielded too high fission barriers. At this point the question arose quite naturally why all effective forces, which have been found very satisfactory for nuclear ground-state properties, led consistently to bad results when extrapolated to the large deformations occurring at the fission barriers [see e.g. ref. ${ }^{65}$ )]. Was this failure linked to some basic deficiency of the effective forces in use or to the Hartree-Fock approach (or to both)? Obviously, in the case of a failure of the interactions in use, one should make sure that one has fully exploited all the available freedom in choosing the force parameters.

Recently, two independent calculations ${ }^{17,24}$ ) using some approximate methods discussed below, showed that the SkM force should lead to a considerably lower fission barrier than was obtained before in Hartree-Fock calculations. The semiclassical results of ref. ${ }^{24}$ ) demonstrated that all the linearly density-dependent

\footnotetext{
+ This holds if one assumes that the pairing matrix element $G$ remains constant as a function of the deformation. Indeed, in the calculation of refs. ${ }^{61,62}$ ) pairing correlations have been included by arbitrarily imposing a pairing gap either constant or varying proportionally to the nuclear surface. A constant $G$ prescription has been later justified by the Hartree-Fock-Bogoliubov results of ref. ${ }^{63}$ ). Such a prescription turns out to be roughly consistent with the uniform gap method used in the self-consistent calculations of the ${ }^{240} \mathrm{Pu}$ fission barrier performed in ref. ${ }^{14}$ ).
} 


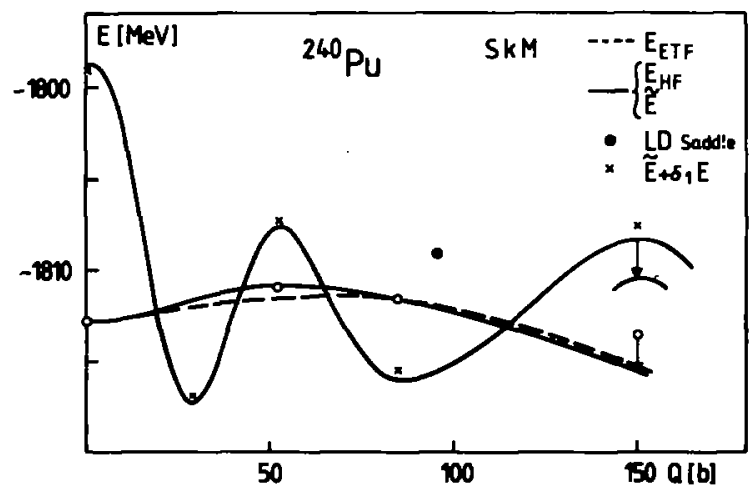

Fig. 8. Self-consistent microscopic ${ }^{240} \mathrm{Pu}$ fission barrier $\left(E_{\mathrm{HF}}\right)$ calculated with the SkM force as a function of the quadrupole moment $(Q)$ of the mass distribution. The self-consistently Strutinsky-averaged energy $(\tilde{E})$, the corresponding first-order shell corrected energy $\left(\tilde{E}+\delta_{1} E\right)$ and the self-consistent extended Thomas-Fermi energy ( $\left.E_{\mathrm{ETF}}\right)$ are also plotted (see fig. 10 caption). The latter has been adjusted to the spherical average energy $\dot{E}$. The self-consistent energies $\left(E_{\mathrm{HF}}\right.$ and $\left.\dot{E}\right)$ calculated at the second fission saddle point have been lowered by $2 \mathrm{MeV}$ as indicated by the arrows to roughly take into account truncation energy effects which become important at such deformations. The circled cross indicates the empirical LDM saddle point ${ }^{6}$ ).

Skyrme forces (SIII-SVI) proposed in ref. ${ }^{2}$ ) lead to almost identical average barriers. Such indications gave us further motivation for calculating with the SkM force the ${ }^{240} \mathrm{Pu}$ deformation energy curve displayed in fig. 8. One immediately notices that the second barrier height which was found at about $20 \mathrm{MeV}$ in previous calculations is now reduced by a factor of two. Applying the same corrections as mentioned before, the barrier will end up by several $\mathrm{MeV}$ too low, as compared with experimental data.

In what follows we will compare quantitatively the results of our self-consistent calculations to those obtained with two approximate methods which will be presented below in some detail.

\subsection{APPROXIMATE METHODS FOR DEFORMATION ENERGIES}

The approach used by Dutta and Kohno ${ }^{17}$ ) to calculate the ${ }^{240} \mathrm{Pu}$ fission barrier has been proposed by Bhaduri et al. ${ }^{15}$ ) for spherically symmetric nuclei. It has been further extended to deformed nuclei in ref. ${ }^{16}$ ). This method approximates the result of the HF iterative process merely from the first two iterations by assuming that

$$
E(\rho)=E\left(\rho^{(1)}\right)+\operatorname{tr}\left[\left(T+V\left(\rho^{(1)}\right)\right)\left(\rho^{(2)}-\rho^{(1)}\right)\right],
$$

where $T$ is the kinetic energy operator. Starting from an ansatz for the mean field, one obtains the density $\rho^{(1)}$ from which the HF hamiltonian $T+V\left(\rho^{(1)}\right)$ is constructed with the eigensolution $\rho^{(2)}$. 


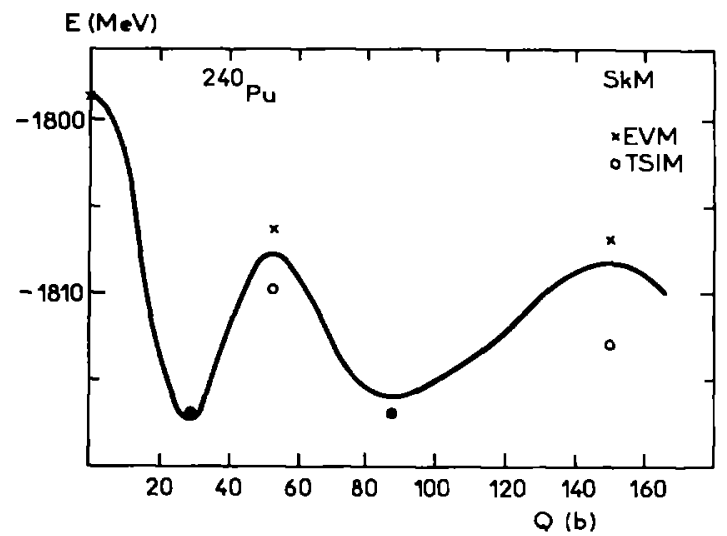

Fig. 9. Self-consistent ${ }^{240} \mathrm{Pu}$ fission barrier as compared with the expectation value method (EVM) and two-step iterative method (TSIM) estimates.

This "two-step iterative method" (TSIM), which has been shown numerically to give remarkable results for spherical and weakly deformed nuclei, turned out to fail for very large deformations as already noted in ref. ${ }^{16}$ ). Indeed it underestimates the self-consistent SkM second fission barrier by about a factor of two as shown in fig. 9, where we have plotted the TSIM estimate ${ }^{17}$ ) for the ${ }^{240} \mathrm{Pu}$ fission barrier calculated with the SkM force. One could object that the incorrect deformation energy behaviour obtained in ref. ${ }^{17}$ ) might be due to the special choice made there of a constant pairing gap $\Delta=\tilde{\Delta}=12 / \sqrt{ } A \mathrm{MeV}$. We have checked that this is not the case by performing a full $\mathrm{HF}+\mathrm{BCS}$ calculation using $\Delta=\tilde{\Delta}$, which turned out, in this case, not to change significantly the deformation energies.

The "expectation value method" (EVM) has been used as an alternative approach for determining self-consistent deformation energy surfaces ${ }^{13,14,66,67}$ ). It consists in using the Slater determinant $\Phi_{w s}$ associated with a reasonable ansatz for the deformed mean field (e.g. Woods-Saxon potential) to calculate the expectation value of the total Skyrme hamiltonian

$$
E_{\mathrm{EVM}}=\left\langle\Phi_{\mathrm{ws}}\left(\beta_{i}\right)\left|T+v_{\text {Skyrme }}\right| \Phi_{\mathrm{ws}}\left(\beta_{i}\right)\right\rangle .
$$

In eq. (7) $\Phi_{\mathrm{ws}}$ depends on one or several deformation parameters $\beta_{\mathrm{i}}$ and $v_{\text {Skyrme }}$ is the Skyrme two-body force. The Slater determinant $\Phi_{\mathrm{ws}}$ is built with singleparticle wave functions which are eigenstates of the one-body hamiltonian

$$
\mathscr{H}_{q}=-\nabla \frac{\hbar^{2}}{2 m_{q}^{*}(r)} \nabla+V_{q}^{\mathrm{ws}}(\boldsymbol{r})-i \nabla S_{q}(\boldsymbol{r})[\nabla \times \sigma], \quad q=\left\{\begin{array}{l}
n \\
p
\end{array}\right\}
$$

which includes an effective mass and a spin-orbit term. In our case the distributions $V_{q}(r), m / m_{q}^{*}(r)$ and $S_{q}(r)$ are Fermi-type functions fitted to the corresponding 
results of a spherical HF calculation. These functions are deformed using a parametrisation used in Strutinsky calculations ${ }^{6}$ ), whose validity for estimating relative energies when using the Skyrme SIII force has been demonstrated in refs. ${ }^{14,66}$ ). We have done similar calculations with the SkM force to calculate the fission barrier of ${ }^{240} \mathrm{Pu}$. As can be seen in fig. 9 the constrained $\mathrm{HF}+\mathrm{BCS}$ curve is reproduced for all deformations to within $1-2 \mathrm{MeV}$ whereas the two-step iterative method fails for deformations beyond the fission isomeric state.

In an alternative approach, one performs self-consistent semiclassical calculations to get average deformation energies of the liquid drop type and smoothly varying mean fields ${ }^{24,32}$ ). From the latter, one may compute the usual Strutinsky shell correction energy. Indeed, numerical calculations have shown ${ }^{12}$ ) that the expansion of the HF energy $E(\rho)$ around the Strutinsky smeared density matrix $\tilde{\rho}$

$$
E(\rho)=E(\tilde{\rho})+\delta_{1} E+\delta_{2} E
$$

converges very rapidly $\left(\delta_{1} E\right.$ is the usual shell correction energy and $\delta_{2} E$ is found to be very small $(<1 \mathrm{MeV})$ if $\tilde{\rho}$ is calculated in a self-consistent way. The equivalence of the semiclassical procedure and the Strutinsky averaging ${ }^{68,69}$ ) allows us therefore to evaluate the full HF fission barrier by adding the shell correction energy $\delta_{1} E$ to the semiclassical deformation energy.

The semiclassical fission barrier by itself allows to estimate in a very economical way the quality of a given Skyrme force at large deformations, since the "experimental" liquid drop fission barrier is indeed well known from Strutinsky calculations ${ }^{6}$ ) (e.g. about $3.5 \mathrm{MeV}$ in ${ }^{240} \mathrm{Pu}$ ). In fig. 8 we show the semiclassical deformation energy curve, obtained for ${ }^{240} \mathrm{Pu}$ with the SkM force. At each value of the quadrupole moment $Q_{2}$, the energy is minimised with respect to the $c$ and $h$ parameters used to deform the density distribution as in ${ }^{6}$ ). The diffuse densities $\rho_{\mathrm{n}}(r)$ nd $\rho_{\mathrm{p}}(r)$ have been assumed to be Fermi-functions across the corresponding liquid drop surface for each deformed shape ${ }^{\dagger}$. We compare the semiclassical fission barrier in fig. 8 with the self-consistently averaged HF barrier obtained as described above. Both curves agree within $\sim 1 \mathrm{MeV}$ for all deformations. This agreement provides a strong confirmation of the relevance of the semiclassical variational method. The latter is only concerned with relative energies. The slight overbinding in the absolute ETF energies has already been discussed in sect. 2 .

Upon adding the shell correction energy $\delta_{1} E$ obtained from a self-consistently Strutinsky averaged HF calculation to the average HF energy at each deformation, the $\mathrm{HF}$ deformation energy curve of ${ }^{240} \mathrm{Pu}$ is reproduced within $\sim 1 \mathrm{MeV}$ as shown in fig. 8. This is another confirmation of the very fast convergence of the Strutinsky energy expansion (8). From this result and the very close agreement of the selfconsistent semiclassical and the self-consistently Strutinsky averaged deformation

\footnotetext{
+ In a former publication ${ }^{24}$ ), the "soft $\pi$ " parametrisation of ref. ${ }^{6}$ ) has been used which, as it turns out, provides significantly higher deformation energies at large deformations. This explains why the SkM barrier given in ref. ${ }^{24}$ ) is higher than the one presented here. [For details, see ref. ${ }^{32}$ ).]
} 
energy curves, it seems clear that applying the Strutinsky shell correction method using the results of semiclassical calculations will give a very satisfactory substitute to the full HF+BCS approach.

\section{Discussion and further possible improvements}

In our present calculations using the effective force parametrisation SkM, we have confirmed the validity of the Strutinsky approach and of the expectation value method, which had been extensively tested earlier with the SIII force. In particular, the good agreement between the self-consistently Strutinsky averaged HF energies and the ones obtained with the variational extended Thomas-Fermi method is worth noting. The two-step iterative method, as practised so far, yields also with the SkM force satisfactory deformation energies up to the fission isomeric state in ${ }^{240} \mathrm{Pu}$, but fails for larger deformations.

The densities calculated with the SkM force for a series of spherical and deformed nuclei lead to charge radii and multipole moments in good agreement with available experimental data. In fact, the agreement is significantly better than it was with previous Skyrme forces (e.g. SIII). However, as we have seen, the SkM force leads consistently to a systematic overbinding and to a too low fission barrier of ${ }^{240} \mathrm{Pu}$. Both these features can be related to a slightly too low surface energy coefficient $a_{\mathrm{s}}$. As shown in fig. 1, a least-square fit of the binding energy excess for the calculated spherical nuclei ${ }^{\dagger}$ indicates that $a_{\mathrm{s}}$ should be increased by $\sim 0.6 \mathrm{MeV}$.

In an attempt to improve the SkM force, we have changed its parameters such as to increase $a_{\mathrm{S}}$, without changing the infinite nuclear matter properties $\rho, E / A$, $K_{\infty}$ and the isoscalar effective mass $m^{*} / m$. Keeping furthermore $x_{1}, x_{2}$ and $x_{3}$ equal to zero (as for SkM), an increase of $a_{\mathrm{S}}$ can only be obtained through an increase of the parameter combination $9 t_{1}-5 t_{2}$ with the combination $3 t_{1}+5 t_{2}$ remaining unchanged. In this way we have determined $t_{1}$ and $t_{2}$ by adjusting the height of the semiclassically calculated fission barrier of ${ }^{240} \mathrm{Pu}$ to its empirical liquid drop (LD) value ${ }^{5-10}$ ). A more detailed discussion of this procedure is given in ref. ${ }^{32}$ ). It leads us to a "modified SkM force" with the parameters $t_{1}=410 \mathrm{MeV} \cdot \mathrm{fm}^{5}$ and $t_{2}=-135 \mathrm{MeV} \cdot \mathrm{fm}^{5}$; all the other parameters are the same as for $\mathrm{SkM}^{3}$ ). The surface energy is then by $0.65 \mathrm{MeV}$ larger than for SkM and is thus consistent with what was estimated above from the binding energy excesses of the SkM force. As seen from table 1 , the relative symmetry energies $J / a_{\mathrm{V}}$ and $\kappa_{\mathrm{s}}$ vary very little between the original and the modified SkM forces. In fig. 10 we present the semiclassical (ETF) fission barriers of ${ }^{240} \mathrm{Pu}$ obtained with both these forces. One notices a close agreement of the "modified SkM" barrier with the empirical LD

\footnotetext{
+ It has been observed fo the SIII force (which has $m^{*} / m \sim \frac{3}{4}$ similarly as SkM), that deformed nuclei are systematically underbound even though the binding energies of spherical nuclei are correct. This feature, as discussed in ref. ${ }^{1}$ ), is related to the existence of ground-state long-range correlations.
} 


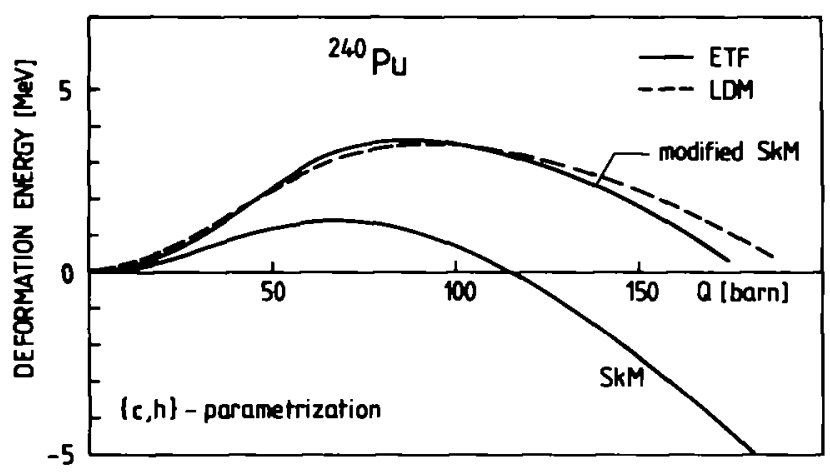

Fig. 10. Self-consistent semiclassical (ETF) ${ }^{240} \mathrm{Pu}$ fission barriers calculated for the SkM and "modified SkM" forces. The liquid drop (LDM) fission barrier is taken from ref. ${ }^{6}$ ). The barriers have been obtained using the $[c, h]$ shape parametrisation of ref. $\left.{ }^{6}\right)$ along the static fission path in the $[c, h]$ plane, and then plotted against the corresponding quadrupole moments.

barrier over a large region of deformations, although only the height of the barrier $(\sim 3.8 \mathrm{MeV}$ relative to the ground state) has been adjusted.

TABLE 7

Same as table 2 for the "modified SkM" force

\begin{tabular}{lrrr}
\hline & $B^{\exp }$ & $B^{\mathrm{HF}}$ & $B^{\text {ETF }}$ \\
\hline${ }^{16} \mathrm{O}$ & 127.6 & 127.7 & 126.0 \\
${ }^{40} \mathrm{Ca}$ & 342.1 & 341.1 & 343.6 \\
${ }^{48} \mathrm{Ca}$ & 416.0 & 420.1 & 419.3 \\
${ }^{56} \mathrm{Ni}$ & 484.0 & 485.4 & 481.0 \\
${ }^{90} \mathrm{Zr}$ & 783.9 & 784.5 & 783.7 \\
${ }^{114} \mathrm{Sn}$ & 971.6 & 969.2 & 972.5 \\
${ }^{132} \mathrm{Sn}$ & 1102.7 & 1110.7 & 1097.8 \\
${ }^{140} \mathrm{Ce}$ & 1172.7 & 1173.9 & 1170.4 \\
${ }^{208} \mathrm{~Pb}$ & 1636.5 & 1636.4 & 1621.9 \\
\hline
\end{tabular}

Using the modified SkM force, we have calculated the binding energy and radii of the same spherical nuclei as considered above, both in the HF+BCS and the extended Thomas-Fermi approach. The results are presented in tables 7 and 8 . As is also illustrated in fig. 11, the experimental binding energies of the $\beta$-stable isotopes are now reproduced to within $\pm 1 \mathrm{MeV}$ by the $\mathrm{HF}+\mathrm{BCS}$ results. For nuclei well off the stability line (such as ${ }^{48} \mathrm{Ca}$ and ${ }^{132} \mathrm{Sn}$ ), the agreement is still rather unsatisfactory. In order to improve on this deficiency, one would have to vary independently the volume and surface asymmetry coefficients. This can be achieved by introducing at least one more non-zero exchange parameter (i.e. $x_{1}, x_{2}$ or $x_{3}$ ). In spite of this defect in the asymmetry energy, it is interesting to note from table 8 that changes in the charge densities, although small, lead consistently 
TABLE 8

Same as table 3 for the "modified SkM" force

\begin{tabular}{lllllllllll}
\hline & $r_{\mathrm{c}}^{\mathrm{exp}}$ & $r_{\mathrm{c}}^{\mathrm{HFC}}$ & $r_{\mathrm{c}}^{\mathrm{HF}}$ & $r_{\mathrm{c}}^{\mathrm{ETF}}$ & $\left(r_{\mathrm{n}}-r_{\mathrm{p}}\right)^{\mathrm{HF}}$ & $\left(r_{\mathrm{n}}-r_{\mathrm{p}}\right)^{\mathrm{ETF}}$ & $\alpha_{\mathrm{n}}$ & $\alpha_{\mathrm{p}}$ & $\rho_{\mathrm{p}}^{0}$ & $\rho^{0}$ \\
\hline${ }^{16} \mathrm{O}$ & 2.73 & 2.73 & 2.81 & 2.78 & -0.03 & -0.02 & 0.448 & 0.452 & 0.0789 & 0.1597 \\
${ }^{40} \mathrm{Ca}$ & 3.49 & 3.48 & 3.52 & 3.47 & -0.05 & -0.04 & 0.460 & 0.468 & 0.0790 & 0.1607 \\
${ }^{48} \mathrm{Ca}$ & 3.48 & 3.48 & 3.54 & 3.54 & 0.16 & 0.17 & 0.500 & 0.442 & 0.0706 & 0.1607 \\
${ }^{56} \mathrm{Ni}$ & 3.75 & & 3.77 & 3.81 & -0.06 & -0.05 & 0.463 & 0.473 & 0.0780 & 0.1592 \\
${ }^{90} \mathrm{Zr}$ & 4.27 & 4.26 & 4.30 & 4.29 & 0.05 & 0.07 & 0.491 & 0.454 & 0.0710 & 0.1574 \\
${ }^{114} \mathrm{Sn}$ & & & 4.60 & 4.61 & 0.08 & 0.08 & 0.496 & 0.453 & 0.0693 & 0.1557 \\
${ }^{132} \mathrm{Sn}$ & & & 4.73 & 4.75 & 0.23 & 0.24 & 0.540 & 0.438 & 0.0621 & 0.1543 \\
${ }^{140} \mathrm{Ce}$ & $4.88 *$ & & 4.89 & 4.89 & 0.13 & 0.14 & 0.512 & 0.446 & 0.0656 & 0.1540 \\
${ }^{208} \mathrm{~Pb}$ & 5.50 & 5.49 & 5.51 & 5.53 & 0.17 & 0.17 & 0.529 & 0.441 & 0.0613 & 0.1504 \\
\hline
\end{tabular}

to an improved agreement with experimental data, compared to the SkM results (table 3).

We would like to emphasize that the modified SkM force parametrisation presented here is by no means a final one. It served here to demonstrate that, indeed, parameter sets can be found which lead to significant improvements of the theoretical predictions of various static nuclear properties, based both on HF+BCS and semiclassical calculations.

To conclude, we may state that, besides the above-mentioned poor reproduction of the binding energies of $\beta$-unstable nuclei, one should try also to include giant resonance and pair correlation properties in the process of refitting the Skyrme force parameters. Investigations along these lines are under way.

Three of us (J.B., C.G. and P.Q.) are indebted to W. Weise and the members of the Institut für Theoretische Physik at the University of Regensburg for their warm hospitality.

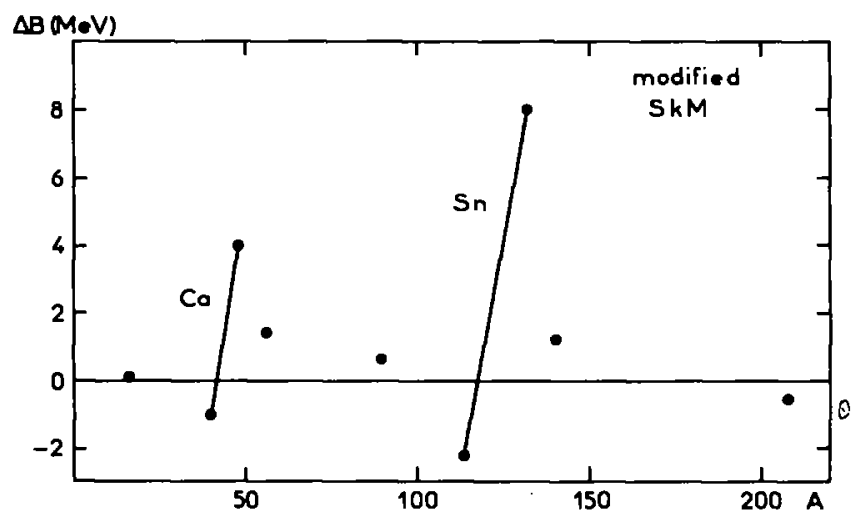

Fig. 11. Binding energy excess $\Delta B$ as in fig. 1 but for the "modified SkM" force. 
Two of the authors (M.B. and H.-B.H.) are grateful to the Institut Laue-Langevin (ILL) for its hospitality and financial support during several visits. All microscopical calculations were performed on the DEC 10 computer at the ILL; we are grateful to the computer staff at the ILL for excellent working conditions.

\section{References}

1) P. Quentin and H. Flocard, Ann. Rev. Nucl. Part. Sci. 28 (1978) 523

2) M. Beiner, H. Flocard, Nguyen Van Giai and P. Quentin, Nucl. Phys. A238 (1975) 29

3) H. Krivine, J. Treiner and O. Bohigas, Nucl. Phys. A336 (1980) 155

4) D. Vautherin, Phys. Rev. C7 (1973) 296

5) V.M. Strutinsky, Yad. Fiz. 3 (1966) 614 [Sov. J. Nucl. Phys. 3 (1966) 499]; Ark. Fys. 36 (1966) 629; Nucl. Phys. A95 (1967) 420; A122 (1968) 1

6) M. Brack, J. Damgaard, A.S. Jensen, H.C. Pauli, V.M. Strutinsky and C.Y. Wong, Rev. Mod. Phys. 44 (1972) 320

7) M. Bolsterli, E.O. Fiset, J.R. Nix and J.L. Norton, Phys. Rev. C5 (1972) 1050

8) S.G. Nilsson, C.F. Tsang, A. Sobiczewski, Z. Szymański, S. Wycech, C. Gustafsson, I.-L. Lamm, P. Möller and B. Nilsson, Nucl. Phys. A131 (1969) 1

9) B.L. Andersen, F. Dickmann and K. Dietrich, Nucl. Phys. A159 (1970) 337

10) D. Scharnweber, W. Greiner and U. Mosel, Nucl. Phys. A164 (1971) 257

11) M. Brack and P. Quentin, Phys. Lett. 56B (1975) 421

12) M. Brack and P. Quentin, Nucl. Phys. A361 (1981) 35

13) C.H. Ko, H.C. Pauli, M. Brack and G.E. Brown, Phys. Lett. 45B (1973) 433; Nucl. Phys. A236 (1974) 269

14) M. Brack, Phys. Lett. 71B (1977) 239

15) A.K. Dutta, R.K. Bhaduri, M.K. Srivastava and M. Vallières, Phys. Lett. $84 B$ (1979) 17

16) A.K. Dutta, M. Vallières, R.K. Bhaduri and I. Easson, Nucl. Phys. A341 (1980) 461

17) A.K. Dutta and M. Kohno, Nucl. Phys. A349 (1980) 455

18) R.K. Bhaduri, Phys. Rev. Lett. 39 (1977) 329

19) M. Durand, M. Brack and P. Schuck, Z. Phys. A286 (1978) 381;

M. Durand, P. Schuck and M. Brack, Z. Phys. A296 (1980) 87

20) J. Bartel, R.K. Bhaduri, M. Brack, M. Durand and P. Schuck, to be published;

J. Bartel and M. Vallières, Phys. Lett. B, in press

21) Y.H. Chu, B.K. Jennings and M. Brack, Phys. Lett. 68B (1977) 407

22) B. Grammaticos and A. Voros, Ann. of Phys. 123 (1979) 359; 129 (1980) 153

23) C. Guet and M. Brack, Z. Phys. A297 (1980) 247

24) C. Guet, H.-B. Håkansson and M. Brack, Phys. Lett. 97B (1980) 7

25) M.J. Giannoni and P. Quentin, Phys. Rev. C21 (1980) 2076

26) N. Marty et al, in Proc. Int. Symp. on highly excited states in nuclei, Jülich (1975) p. 17; D.H. Youngblood et al., Phys. Rev. Lett. 39 (1977) 1188

27) J.P. Blaizot, D. Gogny and B. Grammaticos, Nucl. Phys. A265 (1976) 315

28) X. Campi and D.W.L. Sprung, Nucl. Phys. A194 (1972) 401;

S. Coon and H.S. Köhler, Nucl. Phys. A231 (1974) 95

29) H.v. Groote, E.R. Hilf and K. Takahashi, At. Data and Nucl. Data Tables 17 (1976) 418;

W.D. Myers, At. Data and Nucl. Data Tables 17 (1976) 411;

J.M. Pearson, private communication

30) W.D. Myers and W.J. Swiatecki, Ann. of Phys. 55 (1969) 395; 84 (1974) 186

31) M. Brack, C. Guet, H.-B. Håkansson, A. Magner and V.M. Strutinsky, Proc. 4th Int. Conf. on nuclei far from stability, Helsingør (1981) CERN-Report 81, vol. 1, p. 165

32) C. Guet, H.-B. Hákansson and M. Brack, Nucl. Phys. A, to be submitted

33) D. Vautherin and D.M. Brink, Phys. Rev. C5 (1972) 626

34) A.H. Wapstra and K. Bos, At. Data and Nucl. Data Tables 19 (1977) 177 
35) P. Quentin, in Nuclear self-consistent fields, ed. G. Ripka and M. Porneuf (North-Holland, Amsterdam, 1975) p. 297

36) W. Bertozzi, I. Friar, J. Heisenberg and J.W. Negele, Phys. Lett. $41 B$ (1972) 408

37) R. Engfer et al., At. Data and Nucl. Data Tables 14 (1974) 509

38) I. Sick and J.S. MacCarthy, Nucl. Phys. A150 (1970) 631;

R.F. Frosh et al., Phys. Rev. 174 (1968) 174;

L.A. Fajardo, J.R. Ficenec, W.P. Trower and I. Sick, Phys. Lett. 37B (1971) 363

39) H.S. Köhler, Nucl. Phys. A258 (1976) 301

40) F. Tondeur, J. of Phys. G5 (1979) 1189

41) J. Damgaard, H.C. Pauli, V.V. Pashkevich and V.M. Strutinsky, Nucl. Phys. A135 (1969) 432

42) H. Flocard, P. Quentin, A.K. Kerman and D. Vautherin, Nucl. Phys. A203 (1973) 433

43) D. Gogny, in Proc. Int. Conf. nucl. phys., ed. J. de Boer and H.J. Mang (North-Holland, Amsterdam, 1973) vol. 1, p. 48; and in Nucl. self-consistent fields, ed. G. Ripka and M. Porneuf (North-Holland, Amsterdam, 1975) p. 333

44) F. Cooper et al., Phys. Rev. C13 (1976) 1083

45) H.J. Wollersheim et al., Phys. Lett. 48B (1974) 323

46) H. Flocard, P. Quentin and D. Vautherin, Phys. Lett. 46B (1973) 159

47) M. Girod, private communication

48) J.W. Negele and G. Rinker, Phys. Rev. C15 (1977) 1499

49) J.W. Negele and D. Vautherin, Phys. Rev. C5 (1972) 1472

50. J.W. Negele and D. Vautherin, Phys. Rev. C11 (1975) 1031

51) P.H. Stelson and L. Grodzins, Nucl. Data Tables A1 (1965) 21

52) F.K. McGowan et al., Phys. Rev. Lett. 27 (1971) 1741;

C.E. Bemis, Jr. et al., Phys. Rev. C8 (1973) 1466

53) D. Habs, V. Metag, H.J. Specht and G. Ulfert, Phys. Rev. Lett. 38 (1977) 387

54) U. Götz, H.C. Pauli, K. Alder and K. Junker, Nucl. Phys. A192 (1972) 1

55) M. Brack, T. Ledergerber, H.C. Pauli and A.S. Jensen, Nucl. Phys. A234 (1974) 185

56) P. Möller, S.G. Nilsson and J.R. Nix, Nucl. Phys. A229 (1974) 292

57) F.A. Gareev, S.P. Ivanova and V.V. Pashkevich, [Yad. Fiz. 11 (1970) 1200]; Sov. J. Nucl. Phys. 11 (1970) 667

58) D.W.L. Sprung, S.G. Lie, M. Vallières and P. Quentin, Nucl. Phys. A326 (1979) 37

59) C. Gustafsson, I.-L. Lamm, B. Nilsson and S.G. Nilsson, Ark. Fys. 36 (1967) 613

60) J. Libert, M. Meyer and P. Quentin, Phys. Lett. 95B (1980) 175

61) H. Flocard, P. Quentin, D. Vautherin and A.K. Kerman, in Physics and chemistry of fission 1973, vol. 1 (IAEA, Vienna, 1974), p. 221

62) H. Flocard, P. Quentin, D. Vautherin, M. Vénéroni and A.K. Kerman, Nucl. Phys. A231 (1974) 176

63) J.F. Berger and M. Girod, in Physics and chemistry of fission 1979, vol. 1 (IAEA, Vienna, 1980) p. 265 ; and private communication

64) D. Kolb, R.Y. Cusson and H.W. Schmitt, Phys. Rev. C10 (1974) 1529

65) M. Brack, in Physics and chemistry of fission 1979, vol. 1 (IAEA, Vienna, 1980) p. 227

66) M. Brack, P. Quentin and D. Vautherin, in Super heavy elements, ed. M.A.K. Lodhi (Pergamon, New York, 1978) p. 309

67) F. Tondeur, Nucl. Phys. A338 (1980) 77

68) M. Brack and H.C. Pauli, Nucl. Phys. A207 (1973) 401

69) B.K. Jennings, Ph.D. thesis, McMaster Univ. (1976) unpublished;

B.K. Jennings, R.K. Bhaduri and M. Brack, Nucl. Phys. A253 (1975) 29 